\title{
Physical properties and influence of the tempering on electric properties films $\mathrm{Bi}_{2} \mathrm{Te}_{3}$
}

\author{
Musaver Musayev ${ }^{1}$, Sedreddin Axmedov ${ }^{2}$, Gurban Axmedov ${ }^{2}$ \\ ${ }^{1}$ Department of Physics, Azerbaijan State Oil Academy, Baku, Azerbaijan \\ ${ }^{2}$ Institute of Physics of Azerbaijan NAS, Baku, Azerbaijan
}

Email address:

m_musaver@yahoo.com (M. Musayev), ahmedov_sedi@hotmail.com (S. Axmedov), exmedovqurban@rambler.ru (G. Axmedov)

\section{To cite this article:}

Musaver Musayev, Sedreddin Axmedov, Gurban Axmedov. Physical Properties and Influence of the Tempering on Electric Properties Films $\mathrm{Bi}_{2} \mathrm{Te}_{3}$. International Journal of Materials Science and Applications. Vol. 3, No. 3, 2014, pp. 111-115.

doi: $10.11648 /$ j.ijmsa.20140303.17

\begin{abstract}
Processes of oxidation in the films $\mathrm{Bi}_{2} \mathrm{Te}_{3}$ used in the various thermoelectric film devices working on air at various temperatures are investigated. Samples $\mathrm{Bi}_{2} \mathrm{Te}_{3}$ of $\mathrm{p}$-type thickness received 0,5-1,5 microns a method of discrete evaporation with additional homogenization of a steam phase. Changing technological conditions to raise dust and tempering, have received samples with a wide set of concentration of carriers of a charge from $5 \times 10^{18}$ up to $6 \times 10^{19} \mathrm{~cm}^{-3}$. The fact of formation oxide layers proves to be trues the data electronography the analysis. The electronogram tempering samples alongside with dot reflexes from monocrystal blocks have the continuous polycrystalline rings adequate to a phase of structure $\mathrm{Bi}_{2} \mathrm{O}_{3}$. The method of diffraction electron with high energy - electrongraphy investigates formation of phases in the film condition, received by joint evaporation the components of system $\mathrm{Bi}-\mathrm{S}$. Experimentally investigated dependence of the near nuclear order in amorphous layered $\mathrm{Bi}_{2} \mathrm{~S}_{3}$ from conditions of reception of thin layers - besieged as under influence on molecular pair an external electric field intensity $3000 \mathrm{Vxcm}^{-1}$, and outside of a field. It is established, that internuclear distances and radiuses of coordination spheres in layered, received in conditions of influence of an electric field, are a little shortened in comparison with those without influence of a field.
\end{abstract}

Keywords: Structure, Oxide Layers, Component, Monocrystal, Surface, Electric Field

\section{Introduction}

Need to study the oxidation processes in the films $\mathrm{Bi}_{2} \mathrm{Te}_{3}$ p-type conductivity is related to their application in various film thermoelectric devices (current sources and receivers infrared radiation) operating in air at various temperatures [1].

Samples $\mathrm{Bi}_{2} \mathrm{Te}_{3}$ p- type thickness 0.5-1.5 micrometers were obtained by evaporation of the discrete extra homogenisation vapour phase $[2,3]$. They were a block single crystals (size blocks $\sim 1$ micron) with $\{0001\}$ planes parallel to the substrate surface during the crystallization of them cleaved mica - muscovite vita. $\mathrm{KCl}$ substrate prepared polycrystalline samples (block size $\sim 1000 \mathrm{~A}$ ). After deposition, the film was annealed in an inert atmosphere for several hours. By varying the process conditions and deposition annealing received samples with a wide range of concentrations of carriers (as $5 \times 10^{18}$ to $6 \times 10^{19} \mathrm{~cm}^{-3}$ ). For studying the influence of oxidation on the electrical properties of these films were annealed samples in the isothermal chamber in the air and atmosphere of spectrally pure oxygen. It has been found that films of p-type Bi2Te3 maximum allowable temperature annealing in air is $\sim 600 \mathrm{~K}$. At high temperatures the material is rapidly oxidized and evaporates with the substrate. Therefore, detailed studies have led to the annealing temperatures $580 \mathrm{~K}$. After annealing, the most thick samples $(1-1.5 \mathrm{~m})$ at $580 \mathrm{~K}$ does not change the coefficients of the thermo- electromotive force, Hall, compared to baseline values in the entire investigated range of $77-400 \mathrm{~K}$.

\section{Data Collection and Analysis}

At the same time, the conductivity gradually decreases with increasing annealing time, while a relative decrease in the electroconductivity as compared with the initial value remained constant in the range of 77 - 400K (Fig. 1). Such a decrease in conductivity is not associated with a decrease in the concentration of charge carriers can be caused by the 
formation of a high-resistivity bulk of the film layers of oxides on the block boundaries. In this case, since the coefficient of thermal electromotive force remained constant in these samples, the oxide layer is not formed over the entire thickness of the film, but only in its surface region. We can also assume that the samples cracked during annealing due to the formation of stresses caused by the difference in linear expansion coefficients of the film and substrate. However, the reason for the latter is unlikely, since even during the preparation of the film were annealed in an inert environment at a higher temperature. Moreover, the nature of relationships obtained for films on mica and $\mathrm{KCl}$ is almost identical, despite the significant difference in the coefficients of thermal expansion of mica and $\mathrm{KCl}$.

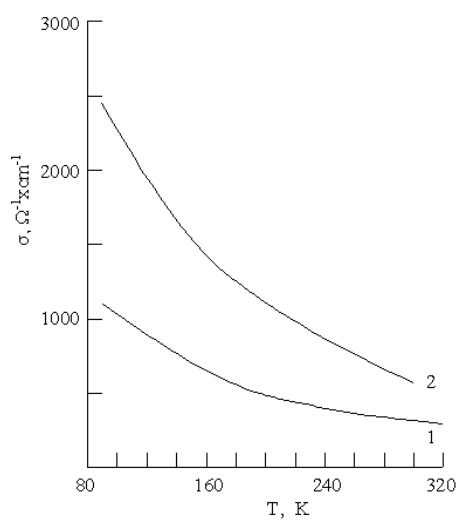

1 - before annealing

2 - after annealing at $580 \mathrm{~K}$ for $5 \mathrm{~h}$.

Figure 1. Temperature dependence of the conductivity of films $\mathrm{Bi}_{2} \mathrm{Te}_{3}$ p-type (1 $\mu$ m thick)

To clarify the nature of the formation of oxide layers were investigated thin $(0.1-0.2 \mu \mathrm{m})$ samples annealed in air at $580 \mathrm{~K}$. We measured the temperature dependences of the thermo- electromotive force $(\alpha)$, Hall $(\mathrm{RH})$ and conductivity $(\sigma)$, in the temperature range $77-300 \mathrm{~K}$.

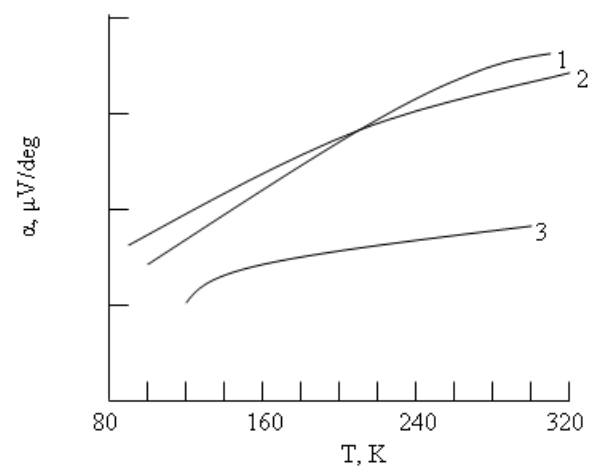

1 - film on mica before annealing

2 - after annealing at $580 \mathrm{~K}$ for $0.5 \mathrm{~h}$.

3 - after annealing at $580 \mathrm{~K}$ for $5 \mathrm{~h}$.

Figure 2. Temperature dependence of the thermo- electromotive force $\mathrm{Bi}_{2} \mathrm{Te}_{3}$ films of p-type (thickness 1,4 $\mu \mathrm{m}$ )

As can be seen from figure 2 short annealing leads to a decrease in the thermal- electromotive force only at temperatures close to room temperature. At longer annealing thermal- electromotive force significantly reduced over the entire range of temperatures. The nature of the dependencies for the films on mica and $\mathrm{KCl}$ does not differ from each other.

At the same time, short-term annealing of the electrical conductivity (fig. 1) is reduced over the entire temperature range. In this case it is manifested most sharply decrease the temperature of liquid nitrogen. A more extended period of annealing conductivity becomes almost constant over the entire temperature range. Such a decrease in electrical conductivity and thermo- electromotive force not accompanied by a change in carrier concentration, as evidenced by the magnitude of the Hall coefficient (fig. 3) do not differ from baseline at $77 \mathrm{~K}$. In this case, after annealing, a decrease of the Hall coefficient with increasing temperature.

Such a change in the temperature dependences of these coefficients may be due to the fact that the oxide layer formed over the entire thickness of the film, have the opposite n-type conductivity in comparison with the conductivity of the base material. Moreover, the carrier concentration in this interlayer is increased sharply with increasing temperature. This leads to a decrease in the coefficients of thermal- electromotive force and Hall with increasing temperature.

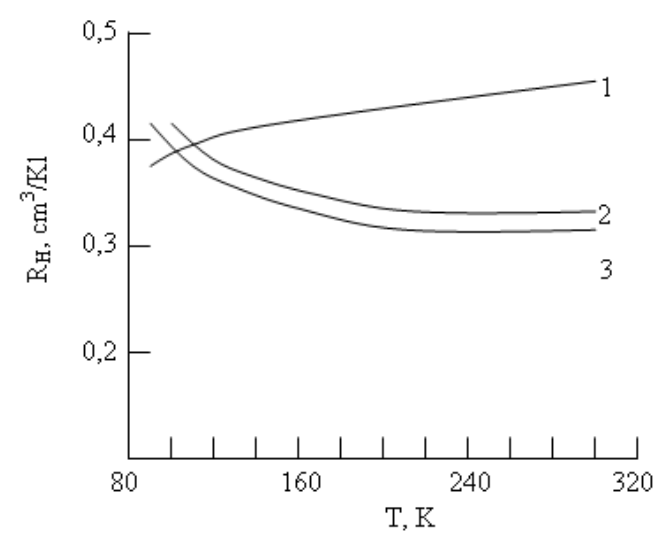

1 - film on mica before annealing

2 - after annealing at $580 \mathrm{~K}$ for $0.5 \mathrm{~h}$

3 - after annealing at $580 \mathrm{~K}$ for $5 \mathrm{~h}$.

Figure 3. Temperature dependence of the Hall coefficient $\mathrm{Bi}_{2} \mathrm{Te}_{3}$ films of p-type (thickness $1.5 \mu \mathrm{m}$ )

The fact of the oxide layer was confirmed by electron diffraction analysis. Electron program annealed samples along with point reflections from single-crystal blocks are solid polycrystalline rings corresponding phase of $\mathrm{Bi}_{2} \mathrm{O}_{3}$.

The assumption that the formation of the matrix precursor oxide phase confirmed by a study of the magnetoresistance.

Due to the fact that the formation of oxide layers resulting in a sharp increase in the resistance of the sample that most significantly at low temperatures, it is possible for thicker specimens to evaluate the thickness of the oxide layer to change the resistance at these temperatures [4]. For films in which the coefficient of thermo- - electromotive force after 
annealing remains constant, conductivity involves only non-oxidized region. Figure 3 shows the dependence of the thickness of the oxide layer on the annealing time at different annealing temperatures. On the basis of these overall dependency of diffusion coefficient of oxygen in the layer forming the oxide films $\mathrm{Bi}_{2} \mathrm{Te}_{3}$ p- type. As is known $D=d^{2} / 16 \sigma$, in the investigated range $470-580 \mathrm{~K}$, the diffusion coefficient varies from $1,6 \times 10^{-17}-5,2 \times 10^{-15}$ $\mathrm{cm}^{2} / \mathrm{sec}$.

\section{Results and Discussion}

In materials science great attention halogen film materials, which can be implemented and amorphous super lattice condition providing a range of useful properties of high hardness in combination with good corrosion resistance and conductivity $[5,6]$. Structure and properties of any substances that are of practical interest, are largely determined by the method of their preparation. Since the phase composition and physical properties of amorphous or single-crystal films prepared by vacuum evaporation, may differ from those of bulk single-crystal glasses massive appropriate formulations, that due to differences in the methods of preparing the compounds of $\mathrm{Bi}-\mathrm{S}$ systems and methods of interpretation of experimental results difficult to compare data from different authors.

Microscopic and X-ray studies as well as from the study of the electrical resistivity, thermoelectric power, magnetic susceptibility, Hall effect and hardness, the existence of phase composition of $\mathrm{Bi}_{2} \mathrm{Te}_{3}$ with a wide range of homogeneity of this phase. In alloys of Bi-Te solid solution except formed by one of the components found in the cubic phase $\mathrm{Bi}_{2} \mathrm{Te}_{3}$ type $\mathrm{BiTe}$ as $\mathrm{NaCl}$ with $\mathrm{a}=0.647 \mathrm{~nm}$. All alloys of $\mathrm{Bi}$-Te alloys are of variable composition, in connection with which the receipt and investigation of thin film samples representing microstructure objects with them unattainable in bulk samples of valuable physical properties of the compounds are characterized by the need to study the system in the process of heat treatment and are determined by scientific interest in aspect of extending the scope of the compounds of this system as infrared detectors, power supplies, autonomous systems and space technology.

With increasing film thickness and substrate temperatures texturing broken. The condensation $\mathrm{Bi}_{2} \mathrm{Te}_{3}$ on the substrate $\mathrm{NaCl}$, located at room temperature, the formed fine-grained polycrystalline structure. Laser deposition of $\mathrm{Bi}_{2} \mathrm{Te}_{3}$ on mica at $623 \mathrm{~K}$ led to the strict orientation of crystallites of the type (0001) $\mathrm{Bi}_{2} \mathrm{Te}_{3} \|$ (0001) mica.

However, the issues on the basis of the phase formation of compounds of $\mathrm{Bi}-\mathrm{Te}$ is not considered, and the data on phase transitions in thin layers in the works, the bibliography which contains a fairly large number of titles are completely absent.

Most fruitful in addressing issues related to the solubility of the components in the compounds of the system Bi-Te, as well as issues of phase formation and phase transformations in this system can serve as a method of kinematic electron.
In this paper we consider the formation of phases with different substructure as a result of reactions and processes of interaction of nana scale vacuum condensates system $\mathrm{Bi}-\mathrm{Te}$ and the kinetics of phase transitions in thin layers of $\mathrm{Bi}_{2} \mathrm{Te}_{3}$.

To establish conditions for the formation of phases in the $\mathrm{Bi}$-Te system, studies were conducted on films obtained by sequential and simultaneous evaporation in vacuum $\sim 10^{-4} \mathrm{~Pa}$ individual components of bismuth and tellurium from two sources. Sources evaporation lagging from each other at a distance of $120 \mathrm{~mm}$ consisted of tungsten conically wound spirals. Substrates were freshly cleaved $\mathrm{NaCl}$ crystals and amorphous celluloid, are at room temperature. In this case, the condensing plane of the layer formed films $\sim 25 \mathrm{~nm}$ thick. To obtain a complete picture on the phase composition in a wide range of temperatures and concentrations of the samples were studied at different temperatures - ambient and from above. This was done using the kinematic electron by taking a series of isothermal electron diffraction. For such electron-defined phase composition of the sample at any temperature and turned several isothermal sections area "composition - temperature."

Analysis of samples obtained by the binary field showed that condensation formed on the plane a few areas: connection area of $\mathrm{Bi}_{2} \mathrm{Te}_{3}$, the realm of pure face-centered cubic phase - BiTe with periods, and the area of the mixture in the vicinity of pure compounds. Produced by film sources Te, were amorphous, crystallization, which allowed identifying them as thin layers of $\mathrm{Bi}_{2} \mathrm{Te}_{3}$. A considerable length of the phases formed in the plane of condensation may be related to a high mobility of bismuth atoms in a molecular beam during evaporation alignment concentrations as a result of migration to the plane of condensation. Diffraction pattern obtained from the film directly under the sources of $\mathrm{Bi}$ and $\mathrm{Te}$ lines contain pure chemical elements not included in the reaction.

At $573 \mathrm{~K}$ leads to recrystallization of the object and the lines in the electron diffraction field will be sharper - there is a further enlargement of the crystallites.

Annealing of thin layers of $\mathrm{Bi}-\mathrm{Te}$, at $\mathrm{t} \sim 423 \mathrm{~K}$ formed unlike $\mathrm{Bi}_{2} \mathrm{Te}_{3}$ films in the crystalline state leads to additional, the intensities of very weak lines in the electron. The emergence of new lines is probably due to the ordering process, as in this case are stored and main diffraction lines of the initial face-centred cubic lattice. Ordered phase is stable in the temperature range $503-523 \mathrm{~K}$, the intensity of the lines, which increases with increasing heat treatment temperature.

A large number of fundamental results obtained from experiments on phase transformations, ie continuous or discontinuous change in the energy, large or small values of activation energy required to complete the phase transition and its speed are explained by the laws of thermodynamics. Basis for understanding many experimental facts observed in phase transitions are the fact that there are two types of phase transitions, called reconstructive and displacement transition: In the first, two passing one another structure or 
state - the transition of the amorphous to the crystalline state are quite different. In the latter case a different structure from each other by mixing in a small crystal lattice, or a large number of atoms. The complexity of studying the kinetics of crystallization of amorphous films is that often remains unknown mechanism of formation of crystals of the new phase and their further growth, the details of which, however, can be obtained as a result of the establishment of time-temperature dependency of crystallization of thin amorphous layers.

Connection sulphides bismuth have valuable properties, in which combination are thermoelectric, magneto-optical and other properties of the semiconductor and the relevance of studies of the effect of external influences, stimulating structural changes - changes in the structural characteristics of thin amorphous semiconductor films halogen is predetermined with the prospects of making various devices, as well as their applications in optoelectronics [7, 8]. Significant impact on the structure and properties of films of semiconducting compounds having the condition of film formation, the nature and character of high external influences on nana scale thin amorphous layers.

Questions phase formation in the system $\mathrm{Bi}-\mathrm{S}$ in the film state and the order structure of various compounds of this system have not been studied, and the available data in the literature focused primarily on the question of synthesis of individual compounds .

The present work is devoted to electron diffraction studies of condensates system $\mathrm{Bi}-\mathrm{S}$, obtained by evaporation of the joint components establishment of conditions for the formation of films with different substructure and the study of short-range order in amorphous films obtained in the absence of any external influences, and under the impact of the molecular pair electric field intensity $3000 \mathrm{Vxsm}^{-1}$.

The phase composition of the resulting films under simultaneous and sequential deposition of bismuth and sulfur was studied by electron diffraction structural analysis on electron diffraction and mass production of brand EMR-102.

Under simultaneous evaporation is to be understood evaporation, whereby both components begin to vaporize at the same time.

Evaporators were wound tungsten baskets conical profile, located at a distance of $120 \mu \mathrm{m}$ apart and at a height of 60 $\mathrm{mm}$ above the plane of condensation.

These baskets were placed sample Bi and $\mathrm{S}$ at 11.1 and 2.4 $\mathrm{mg}$, respectively.

Evaporation was performed on monocrystalline $\mathrm{NaCl}$, $\mathrm{KCl}$, and celluloid substrates at room temperature. 2 appear on evaporation of molecular flow. Each of molecular flow exists independently from each other, although they penetrate the particles the same volume. At the end of the evaporation on substrates obtain binary field equal to the distance between the sources.

Electron-diffraction analysis formed on the plane of film condensation showed that with simultaneous evaporation of bismuth and sulfur in a fairly wide range plane condensation formed amorphous films with values $S=4 \pi \sin \theta / \lambda=16,7 ; 28.2$; $47.8 \mathrm{~nm}^{-1}$. Crystallization of amorphous films at $323 \mathrm{~K}$ allowed to resume phase of $\mathrm{Bi}_{2} \mathrm{~S}_{3}$. Calculation of electron diffraction from polycrystalline shows that $\mathrm{Bi}_{2} \mathrm{~S}_{3}$ thin films with a thickness of $\sim 25 \mathrm{~nm}$ crystallize based on orthorhombic unit cell with the periods of the crystal lattice of $a=1,12 ; b=1,13 ; c=0,398 \mathrm{~nm}$, which agrees well with the data presented in [9]. By electronogramm obtained from films that are closer to hitch $\mathrm{Bi}$ and $\mathrm{S}$, obtained mainly the same picture, but along with the lines of pure $\mathrm{Bi}_{2} \mathrm{~S}_{3}$ observed diffraction lines $\mathrm{Bi}$ and $\mathrm{S}$. Samples are annealed to $443 \mathrm{~K}$ for 25-30 minutes and subsequent elektronography showed that the phase distribution on the plane condensation has not changed. Lattice parameters $\mathrm{Bi}_{2} \mathrm{~S}_{3}$ remain unchanged. At the edge of $\mathrm{Bi}$ obtained $\mathrm{Bi}+\mathrm{Bi}_{2} \mathrm{~S}_{3}$, and at the edge of $\mathrm{S}$ is obtained $\mathrm{Bi}_{2} \mathrm{~S}_{3}+\mathrm{S}$, i.e. observed excess components not included in the reaction to form other compounds of bismuth sulphide. In a very narrow plane of condensation obtained $\mathrm{Bi}+\mathrm{Bi}_{2} \mathrm{~S}_{3}+\mathrm{S}$.

For sequential deposition of $\mathrm{Bi}, \mathrm{S}$ formed film $\mathrm{Bi}_{2} \mathrm{~S}_{3}$ amorphous at room temperature. The phase composition of the films is different from the case of simultaneous deposition components. As with simultaneous evaporation rated immediately after deposition on the plane formed by the condensation of $\mathrm{Bi}_{2} \mathrm{~S}_{3}$ with excess $\mathrm{Bi}$ and $\mathrm{S}$. The change in the order of deposition of components does not lead to changes in the nature of phase formation.

During the deposition of the synthesized compound $\mathrm{Bi}_{2} \mathrm{~S}_{3}$ on the LiF substrates with $\mathrm{Ts}=363-373 \mathrm{~K}$ also formed polycrystalline films. Recrystallization polycrystalline film at a temperature of $433-443 \mathrm{~K}$ leads to the formation of textured films. In the area of the substrate temperatures of 463 - $473 \mathrm{~K}$, a mixture of polycrystalline sample with mosaic single crystal.

With increasing temperature, the intensity of the substrate diffraction lines corresponding to the polycrystalline, decrease, and point reflections indicating the formation of single-crystal blocks becomes larger. Further increase in temperature up to $423 \mathrm{~K} \mathrm{LiF}$ substrates leads to the formation of a single crystal of high perfection in the electron from which visible spot reflexes.

At higher temperatures, the resulting films monocrystalline disturbed.

Samples useful for the determination of short-range order in amorphous $\mathrm{Bi}_{2} \mathrm{~S}_{3}$ obtained in two ways: joint $\mathrm{Bi}$ and $\mathrm{S}$ deposition from two evaporation sources, and the evaporation of the synthesized compound of the formulation of fresh chips $\mathrm{Bi}_{2} \mathrm{~S}_{3}$ by (100) planes of single crystals of $\mathrm{NaCl}$ and $\mathrm{KCl}$, having a room temperature. The film thickness was $25-30 \mathrm{~nm}$.

Options range order of the atomic structure of amorphous $\mathrm{Bi}_{2} \mathrm{~S}_{3}$ determined by the results of electron-diffraction studies on the device EMR-102 and calculating the radial distribution function of atoms method of integral equations [10-12], which is based on the formula given in [9]. Amorphous films are stable at room temperature and crystallize in the temperature range $423-443 \mathrm{~K}$ for $10-15 \mathrm{~min}$ 
in the rhombic lattice with periods of the unit cell $\mathrm{a}=1.115$; $\mathrm{a}=1.129 ; \mathrm{c}=0.398 \mathrm{~nm}[13]$.

\section{Conclusion}

Thus, we find that on substrates LIF possible formation of $\mathrm{Bi}_{2} \mathrm{~S}_{3}$ samples with different substructure, and obtained for amorphous films of $\mathrm{Bi}_{2} \mathrm{~S}_{3}$ experimental data indicate similarity structures range order in the amorphous and crystalline $\mathrm{Bi}_{2} \mathrm{~S}_{3}$. Covalent bonding force between atoms in the crystal lattice is preserved in the amorphous films.

In amorphous films produced under the impact of a molecular pair of external electric field intensity $3000 \mathrm{Vxsm}^{-1}$ inter atomic distances - a few radii of coordination spheres, be shortened $\left(\mathrm{r}_{1}=0,227 \mathrm{~nm}\right.$, the second - $\mathrm{r}_{2}=0,340 \mathrm{~nm}$, and the third $\left.-\mathrm{r}_{3}=0,420 \mathrm{~nm}\right)$, is a more stable state of the amorphous phase $\mathrm{Bi}_{2} \mathrm{~S}_{3}$. The matrices of amorphous as well as in the case of thin layers deposited are comprised of the electric tetrahedral and octahedral environments atoms.

\section{References}

[1] Guozhen S., Di C., Kaibin T., Yitai Q. // Nanotechnology 15 (2004) 1530-1534.

[2] Ismailov D., Axmedov Q., Shafizade R. // Reports of Academy of Azerbaijan (1989). vol. XLV. no.4. pp. 4. (in Russian)
[3] Abdullaev G., Isgenderov S., Axmedov Q. // Abstracts IV-Union Conference on the Chemistry and Technology of chalcogenides and chalcogen. Karaganda. (1990). pp. 405.

[4] Boykov Yu., Qribanov O., Danilov V. // Inorganic materials (1990). v. 26. no. 8. pp. 1628.

[5] Fillips C. Mutual diffusion and reaction. M., Mir, (1982), p. 576

[6] Skakov Yu., Edneral N., Mazzora X., Kropasin V. N Scientific papers. Moscow, Metallurgy, (1983) p. 8 (in Russian)

[7] Andries M., Ponomar V., Smirnov, Смирнов V, Mironos A. Quantum Electronics, (1986), v.13, no 6, pp.1093-1117

[8] Kalinin S. Ivanichkiy V., Chiminech V., Dovqoshey N. Surface. Physics, chemistry, mechanics, (1984), no 12, p.100-1035.

[9] Boqolyubov N. Problems of dynamical theory in statistical physics. Moscow: Gostekhizdat, (1946) 359p.

[10] Fisher I. Statistical theory of liquids. M. IFML, (1961) 284p.

[11] Tatarinova L. The structure of amorphous solid and liquid substances. Moscow: Nauka, 1983, 151p.

[12] Xajen M., Anderko K. The structure of binary alloys: in 2 volumes, Volume 1, Moscow: Metallurgy, (1962), 608p.

[13] Nabitovich I., Stechiev Ya, Volosuk Ya. Defining a coherent intensity and the background intensity on the experimental curve of electron scattering. Crystallography, (1967), v.12, no 4, pp.584-590. 